\title{
HORA LIBRE, UNA EXPERIENCIA TELEVISIVA HACIA LA EDUCOMUNICACIÓN
}

\author{
Silvana CRIStina IOVAnNa CaISSÓn \\ Consejo Nacional de Investigaciones Científicas y Técnicas (Conicet), \\ Universidad Nacional de San Juan, Argentina / \\ silvanaiovanna@hotmail.com
}

Recibido: 6/5/2019 / Aceptado: 30/5/2019

doi: $10.26439 /$ contratexto2019.n032.4615

Resumen. En el presente artículo analizaremos la experiencia de Hora libre, un programa de televisión realizado por niños, niñas y jóvenes en canales de televisión comunitarios de Argentina entre el 2017 y el 2018, como parte de una serie de políticas públicas que colaboran con el ejercicio del derecho a la comunicación. El tema será abordado desde una perspectiva crítica de la educomunicación, estudios culturales, teorías de la educación popular y estudios sobre políticas comunicacionales en Argentina. Se realizará una primera sistematización de la propuesta a partir del análisis del contexto políticocomunicacional, los actores intervinientes, las prácticas comunicacionales y las lógicas institucionales que se pusieron de manifiesto en el proyecto. Se propone este trabajo como espacio de reflexión para la acción en futuras instancias que vinculen tanto el campo de la comunicación como el de la educación en pos del fortalecimiento de políticas comunicacionales democráticas.

Palabras clave: derecho a la comunicación / educación / políticas públicas / medios comunitarios

\section{HORA LIBRE, A TELEVISION EXPERIENCE TOWARDS EDUCOMMUNICATION}

Abstract. This article analyzes the experience of Hora Libre (Free Time), a television program hosted by boys, girls and young people on Argentine community TV channels between 2017 and 2018, as part of a series of public policies that promote exercising the right to communication. The topic will be approached from a critical perspective

1 Doctoranda en Políticas Públicas y Comunicación por la Universidad Nacional de San Juan, Argentina. https://orcid.org/0000-0002-1926-057X 
of educommunication, cultural studies, popular education theories and studies on communication policies in Argentina. In this way, a first systematization of the proposal will be carried out based on the analysis of the political-communication context, the intervening actors, the communication practices and the institutional logics which became evident in the project. Finally, this work is proposed as a space for reflection to act in future instances that bind the fields of communication and education in pursuit of strengthening democratic communication policies.

Keywords: Communication right / education / public policies / community media

\section{“HORA LIBRE”, UMA EXPERIÊNCIA TELEVISIVA PARA A EDUCOMUNICAÇÃO}

Resumo. Neste artigo analisaremos a experiência de "Hora Libre", um programa de TV realizado por crianças e jovens em emissoras comunitárias de televisão na Argentina entre 2017 e 2018 e que faziam parte das políticas públicas que colaboraram com o exercício do direito à comunicação. A abordagem dessa experiência será feita a partir de uma perspectiva crítica da educomunicação, dos estudos culturais, das teorias da educação popular e dos estudos sobre políticas de comunicação na Argentina. Dessa forma, uma primeira sistematização da proposta será realizada a partir da análise do contexto político-comunicacional, dos atores intervenientes, das práticas comunicacionais e das lógicas institucionais, evidenciadas no projeto. Finalmente, esse trabalho é proposto como um espaço de reflexão para a ação em futuras instâncias que relacionem o campo da comunicação e da educação na procura do fortalecimento das políticas democráticas de comunicação.

Palavras chaves: Direito à Comunicação / Educação / Políticas Públicas / Mídia Comunitária 


\section{INTRODUCCIÓN}

En Argentina, luego de la sanción de la Ley de Servicios de Comunicación Audiovisual, Ley 26.522 (2009), medios de comunicación comunitarios e instituciones escolares llevaron adelante experiencias comunicacionales vinculadas al ejercicio del derecho a la comunicación. Algunas de ellas se centraron en el uso de las tecnologías como herramientas para la libre expresión, otras apostaron por revisar el fundamento de la comunicación y generaron procesos participativos en los que niños, niñas y jóvenes fueron los protagonistas en la acción.

En este contexto, a partir del 2017, se llevó a cabo Hora libre, un proyecto televisivo impulsado por la Defensoría del Público de Servicios de Comunicación Audiovisual, y coordinado con escuelas y medios comunitarios del país. Su propuesta consistió en la realización de un ciclo de programas de televisión cuyo formato atraviese los géneros de entretenimiento, educación y cultura. Así, se diseñó un magacín de tres bloques y cinco secciones. Cada sección contaría con la participación en vivo de niños, niñas y jóvenes de escuelas públicas, pero además con la producción de contenidos audiovisuales relacionados producidos por los estudiantes desde su identidad local e intereses socioculturales.

¿Cómo desarrollar este proyecto bajo un paradigma de protección integral de derechos? ¿Desde qué perspectiva de comunicación y educación pueden trabajar los docentes? ¿Qué rol pueden cumplir en la producción audiovisual que sus estudiantes realicen? ¿Cuál es el lugar de la escuela en estas experiencias comunicacionales? Para abordar estos y otros interrogantes, y a fin de analizar las prácticas que en esta experiencia tuvieron lugar, se tendrán en cuenta diversos aportes: teorías críticas de la comunicación (Martín Barbero, 2002; Hall, 2003; Mata, 2006; Vinelli y Rodríguez Esperón, 2004), la perspectiva pedagógica de la educación popular (Freire, 2002; Huergo, 2015), reflexiones vinculadas a la educomunicación (Arena Fernández, 2012; Barbas Coslado, 2012) y estudios sobre políticas comunicacionales en Argentina (Mastrini y Becerra, 2017; Rossi, 2016).

A lo largo de los tres primeros ejes, describiremos el proceso de surgimiento y desarrollo de Hora libre hasta su primera experiencia en la Ciudad Autónoma de Buenos Aires. Luego, profundizaremos el análisis del programa realizado en la provincia de Mendoza durante el 2018, en Giramundo Televisión Comunitaria, donde la autora del presente artículo tuvo la oportunidad de participar como docente y miembro del canal.

Las herramientas metodológicas utilizadas para describir y analizar esta experiencia se centraron en la lectura de material de archivo y documentos oficiales, visionado de materiales audiovisuales, la realización de entrevistas en profundidad a actores institucionales y la observación participante, como clave en este trabajo. 
Desde una perspectiva epistémica crítica contemporánea, nos proponemos así abordar la participación de jóvenes y adultos en Hora libre como experiencia de educación y comunicación comunitaria, allí donde tienen lugar la expresión, organización y transformación de las representaciones e identidades sociales.

\section{PARADIGMAS COMUNICACIONALES Y EDUCACIÓN MEDIÁTICA EN ARGENTINA ${ }^{2}$}

Desde la vuelta a la democracia en 1983, la disputa por ejercer el derecho a la comunicación en Argentina se tradujo no solo en una lucha jurídica, sino, además, en la puesta en práctica de experiencias educativas, y el sostenimiento y crecimiento de medios de comunicación comunitarios, alternativos y populares, en todo el país.

María Cristina Mata (1988) señala que, luego de la censura impuesta por la última dictadura militar en Argentina (1976-1983), tuvo lugar una "multiplicación de sujetos y lugares de enunciación" (Mata, 1988), y consecuentes experiencias sociales y comunitarias vinculadas con la comunicación y educación popular (Arena Fernández, 2012). Alrededor de tres mil nuevas radios surgieron por esos años (Lamas y Lewin, 1995) y algunas se definieron como comunitarias, alternativas o populares (Vinelli y Rodríguez Esperón, 2004). Asimismo, Roxana Morduchowicz (1999) señala que, durante dicho período, en las escuelas de la ciudad de Buenos Aires se desarrollaron experiencias relacionadas con la lectura reflexiva y crítica de noticias sobre la guerra de Malvinas (1982) y se abordaba así la comunicación desde la libertad de expresión y el fortalecimiento de la ciudadanía.

Ahora bien, los años 90 estuvieron marcados por la profundización del modelo neoliberal, crecimiento de los capitales internacionales y conformación de grandes monopolios empresariales en la Argentina. Tanto las políticas educativas como las comunicacionales viraron hacia la privatización, restringiendo el acceso a bienes y servicios y profundizando las desigualdades en la población (Rossi, 2016). En lo que respecta al campo de las comunicaciones, si bien hubo intentos de promover acciones democratizadoras y poner límites a los monopolios mediáticos por parte de un sector de la ciudadanía, solo se lograron algunas modificaciones a la ley vigente que no tuvieron impacto en el mapa mediático consolidado en el país.

En este contexto, el desarrollo de experiencias educativas y comunicacionales se concentró en la implementación de nuevas tecnologías de la información y de la comunicación (NTIC) en las aulas, principalmente con el ingreso de las salas de computación en las escuelas y el uso del periódico o revista escolar como herramienta de difusión institucional.

2 Para conocer más sobre el desarrollo y actualidad de la educación mediática en Argentina referir a lovanna Caissón, S. y Martino, B. (2018). Educación mediática en Argentina: balance y perspectivas de un campo en construcción. Editorial Routledge, artículo aceptado, en prensa. 
Los primeros años del siglo XXI, organizaciones sociales, políticas, gremiales, educativas, universitarias, entre otras, nucleadas en la Coalición por una Radiodifusión Democrática, organizaron debates públicos por todo el país con el objetivo de difundir la propuesta "21 puntos por una comunicación democrática" (Sel, 2010). Luego de aportes y modificaciones que diversos sectores de la ciudadanía y empresarios realizaron al proyecto de ley, el 10 de octubre del 2009 se sancionó la Ley de Servicios de Comunicación Audiovisual (Ley 26.522, 2009). La nueva normativa representó un cambio importante ya que en ella el ejercicio de las comunicaciones es considerado un derecho humano fundamental.

Se fue instalando así en la escena pública la necesidad de traducir la letra de la ley en políticas públicas que habiliten el ejercicio del derecho a la comunicación ${ }^{3}$, y al cumplimiento de otros derechos a través de este. Se renovó el debate en las escuelas sobre la comunicación y la educación, pero esta vez a partir de profesionales de la comunicación que, como licenciados y profesores, pudieron acceder al sistema educativo.

Dos ejes se trabajaron en este sentido. Por un lado, el uso de las NTIC (nuevas tecnologías de la información y de la comunicación) y el potencial educativo que representan, sobre todo a partir del uso masivo de los teléfonos celulares con acceso a internet. El debate renovó posiciones en torno al determinismo tecnológico o, como afirma Orozco Gómez (1986), dicha falacia debe combatirse con racionalidad pedagógica. Por otro lado, se hizo hincapié en el respeto integral de los derechos mediante el ejercicio del derecho a la comunicación, lo que visibilizó debates en torno a proyectos educativos, el rol docente y el abordaje de la comunicación en la educación.

Respecto a esto último, la Ley SCA explicita acciones para el respeto de los derechos de niños, niñas y adolescentes (Ley 26061, 2005) que deben ser tenidas en cuenta por medios masivos hegemónicos y por medios comunitarios, alternativos, populares, y docentes que quieran articular con estos.

3 El derecho a la comunicación es un concepto superador del de libertad de prensa y del derecho a la información (Mastrini, G. y De Charras, D., 2004), y comienza a ser utilizado a partir de la Conferencia General de la Unesco en Nairobi 1976. Argentina adhirió a dicho Convenio mediante la Ley 23.478 de 1986, adquiriendo así rango constitucional. Esta convención definió la comunicación como un "proceso de interacción social, a través de un intercambio equilibrado de información y experiencia [...]. El objetivo es el de alcanzar un sistema de comunicación horizontal, basado en una distribución equitativa de los recursos y de las facilidades, que permita a todas las personas enviar y recibir mensajes" (Graziano, 1980, p. 71). En la Carta de Belgrado de 1977 se acordaron los indicadores fundamentales para la democratización de las comunicaciones en función de la implementación de políticas nacionales de comunicación: el acceso y la participación. El acceso implica la posibilidad de que el público se acerque a los sistemas de comunicación, mediante la elección y la comunicación de retorno; la posibilidad de elección de productos diversos, es decir, que en todo el territorio exista la diversidad de programación más allá de si se pueden formar mercados o no, evitando la conformación de monopolios mediáticos. La participación implica la posibilidad de la población de intervenir en la producción de mensajes, en la toma de decisiones y en la contribución para la formulación de planes y políticas de comunicación masiva (Rossi, 2016). 
En el artículo 17 de la Ley SCA se propone la creación del Consejo Asesor de la Comunicación Audiovisual y la Infancia, que establece, entre otras funciones, mecanismos de análisis y estudios de medios que permitan mejorar la calidad en los contenidos audiovisuales para este sector de la población. En el artículo 68, se fija una serie de pautas de protección de la niñez ante los contenidos que puedan ofenderla o dañarla, como horarios de protección en la programación. El artículo 153, en el inciso g, dispone un fondo de financiamiento para la producción de programas de televisión "de calidad para niños, niñas y adolescentes" (Ley 26.522, S/R 2009). El artículo 71 advierte que los medios de comunicación son responsables de velar por el cumplimiento de varias normativas, entre ellas la Ley 26.061, y, en ese sentido, el artículo 81 recomienda evitar estereotipos discriminatorios en publicidades que impliquen a la niñez y la juventud como protagonistas y destinatarios. Asimismo, se pensó en la generación de espacios de acceso y participación a medios de comunicación, por lo que se otorgaron, por el artículo 149, licencias de radiodifusión a escuelas públicas estatales en todo el país ${ }^{4}$.

\section{DERECHO A LA COMUNICACIÓN, DE LA LEY A LA ACCIÓN: EL ROL DE LA DEFENSORÍA DEL PÚBLICO}

Ahora bien, como venimos desarrollando, la Ley SCA abrió una nueva etapa para la democratización de las comunicaciones promovió la educación y el respeto hacia las audiencias a través de la creación de la Defensoría del Público de Servicios de Comunicación Audiovisual ${ }^{5}$. Este organismo autárquico tiene diversas áreas de trabajo: protección de derechos y asuntos jurídicos, análisis, investigación y monitoreo, comunicación institucional, capacitación y promoción, y relaciones con las audiencias ${ }^{6}$.

Dentro de las funciones principales del organismo se encuentra la recepción y procesamiento de denuncias realizadas por las audiencias que perciben sus derechos comunicacionales vulnerados en los medios audiovisuales. Asimismo, realiza audiencias

4 La Autoridad Federal de Servicios de Comunicación Audiovisual (AFSCA), creada a partir de la Ley SCA en el 2010, adjudicó 280 licencias de radio FM para escuelas y otras 10 a universidades nacionales (Espada, 2017). Asimismo, la política fue acompañada hasta 2015 por la creación del Programa Nacional de Extensión Educativa-Centro de Actividades Juveniles (CAJ), equipando las radios y empleando docentes que puedan gestionarla y trabajar junto a los estudiantes de las mismas escuelas beneficiadas. Desde el cambio de gobierno, el 10 de diciembre del 2015, los programas y las radios se encuentran sin financiamiento $y$, en pocos casos, funcionando en el marco del trabajo escolar de los docentes de planta. Desde el Ministerio de Educación se respaldó el crecimiento y sostenimiento de estas nuevas radios desde el Programa de Radios Escolares, mediante el cual se consiguió el equipamiento necesario y se contrataron docentes capacitadores en radio y coordinadores de proyectos.

5 Si bien la reglamentación de la Ley SCA se efectivizó en agosto del 2010, a través del artículo 19 se creó en el 2012 la Defensoría del Público de Servicios de Comunicación Audiovisual.

6 Para más información sobre el funcionamiento de la Defensoría del Público de Servicios de Comunicación Audiovisual, consultar su sitio web. 
públicas anualmente en las que miembros de comunidades, organizaciones y escuelas pueden contar sus experiencias como audiencias, denunciar agravios y hacer recomendaciones para el diseño de políticas públicas.

Desde la Dirección de Capacitación y Promoción, la defensoría ha generado toda una línea de trabajo vinculada a la promoción de los derechos comunicacionales con escuelas, organizaciones sociales, grupos de disidencia sexual, comunidades indígenas y medios de comunicación comunitarios. En este marco, se desarrollan talleres de capacitación con docentes y estudiantes sobre los derechos comunicacionales de niños y adolescentes, y proyectos vinculados con su participación en medios audiovisuales.

Desde el 2012, el organismo fue promotor de diversas experiencias radiofónicas y audiovisuales en la que niños niñas y jóvenes pudieron expresarse "pero desde su realidad y no a través de la realidad de los otros" (Montoya, como se citó en Arenas Fernández, 2012, p. 13). Para ello, desde el área de capacitación se implementa el trabajo coordinado, colectivo y desde una perspectiva de la educación popular.

Cecilia Uriarte, coordinadora de Niñez, Juventud y Medios, de la Dirección de Capacitación y Promoción de la Defensoría del Público, explica que dicha área trabaja para promover el reconocimiento de los derechos comunicacionales a través de capacitaciones (Uriarte, comunicación personal, 27 de octubre del 2018). Asimismo, los talleres para docentes encuentran su fundamento en que "la escuela es el espacio donde se puede dar con mayor facilidad e igualdad el acceso al conocimiento de los derechos umanos" (comunicación personal, 27 de octubre del 2018).

En todas esas instancias, la defensoría trabaja desde la perspectiva de la educación popular implementando técnicas relacionadas con los abordajes de Paulo Freire (2009). Para este pedagogo, enseñar exige respeto a los saberes de los educandos, pero, además, exige "discutir con los alumnos la razón de ser de esos saberes en relación con la enseñanza de los contenidos" (Freire, 2009, p. 31).

Así, desde el 2014 se promovieron capacitaciones en escuelas que fundamentaron el uso de herramientas radiofónicas como parte del derecho a la comunicación. Además, se incentivó a los estudiantes a participar en las audiencias públicas regionales ${ }^{7}$

7 Durante 2014, se realizaron en todo el país siete audiencias públicas en diferentes regiones. En ellas participaron " 1750 chicas y chicos, casi mil fueron oradores/as, representaron a 220 organizaciones, escuelas y otros espacios de pertenencia de las 24 provincias argentinas. Este proceso fue acompañado por 170 instancias de formación y de debate en las que participaron 4000 jóvenes" (Defensoría del Público de Servicios de Comunicación Audiovisual, 2014). A partir de esto, el organismo generó una declaración sobre la juventud y los medios audiovisuales que está disponible en su página web y en el canal de YouTube de la defensoría hay fragmentos de estas ponencias. 
y en espacios en las radios comunitarias que integran la red $\mathrm{FARCO}^{8}$. Por otro lado, se llevó adelante Aire joven, un boletín radial realizado enteramente por estudiantes de varias provincias de la Argentina, coordinando la producción y difusión de diversos talleres de radios escolares.

En el mismo sentido, pero en otro soporte, se produjo Hora libre, un magacín televisivo en el que estudiantes ponen en pantalla lo que viven en sus escuelas y barrios, opinan y visibilizan sus intereses a través de las televisoras comunitarias del país y sus canales de YouTube.

Estas experiencias invitan a nuevas reflexiones en el campo de la comunicación y la educación en Argentina, puesto que el rol de la defensoría es lograr encontrar un punto de encuentro entre la cultura escolar y la cultura mediática (Huergo, 1997). Jesús Martín Barbero (1996) ha reflexionado acerca de estas dos culturas y apunta la dimensión temporal como factor clave en la crisis de la escuela frente a los nuevos modos de sentir, pensar y producir a partir de la cultura mediática.

Sobre cada una de las capacitaciones, el organismo genera instancias que van desde la reflexión y el análisis crítico a la posibilidad de producción. El eje es producir nuevos contenidos evitando la reproducción de las representaciones sociales que se construyen en los medios masivos y hegemónicos sobre niños, niñas y jóvenes de diferentes sectores sociales.

Por ello, al inicio de cada taller, se brindan datos extraídos de los informes del monitoreo de noticias realizados anualmente por la defensoría, a partir de los cuales se ha podido visibilizar la reiteración de representaciones de niños y jóvenes vinculados con la pobreza, el peligro y la inseguridad" (Defensoría del Público de Servicios de Comunicación Audiovisual, 2018). Al respecto, Rosana Reguillo Cruz (2000) explica que la operación de los medios inscribe sobre los cuerpos de los jóvenes un imaginario que los vincula a la delincuencia, y que el estigma que pesa sobre ellos justifica la vigilancia y el

8 FARCO (Foro Argentino de Radios Comunitarias) es una organización que agrupa radios populares y comunitarias de la República Argentina. Surge a principios de los años 90 y actualmente cuenta con 91 asociadas en todo el territorio argentino.

9 De acuerdo con el “Informe: 5 años de monitoreo de noticias (2013-2017)" (Defensoría del Público, 2018, p. 36), "en todos los monitoreos realizados, Niñez y adolescencia / Juventud fue consignado como tópico secundario más veces que como tópico prevalente. [...] prácticamente no hay noticias -o cuando menos, un conjunto relevante de noticias- que aludan a derechos de niñas, niños y adolescentes, a acceso o demandas de la niñez, a acciones sociales que trasciendan el campo delictual (como víctimas o como victimarios). Si bien puede considerarse una relación forzada o fetichizada, resulta relevante la ausencia de vínculos noticiosos entre Niñez y adolescencia con tópicos como Educación; Salud / Discapacidad; Ambiente; Deportes y con Política. La invisibilización y policialización de la juventud fue uno de los primeros hallazgos de esta investigación desde el 2013. Su ratificación año a año ha mostrado que esta forma de noticiabilidad es estructural y estructurante del sistema". 
control social, ubicando en la sección Policiales muchas de las noticias sobre jóvenes y delincuencia. Luego, mediante actividades y juegos, se van desarrollando relatos desde las subjetividades que vivencian los talleres, y que luego serán parte de producciones audiovisuales o radiales.

El trabajo educativo de la defensoría no se remite únicamente a las audiencias (y en el presente caso en la comunidad educativa). A partir de la presentación de los informes y de las denuncias que las audiencias realizan a través de la página web del organismo, se realizan capacitaciones y acuerdos de revisión de contenidos con productoras y periodistas de los medios comerciales más importantes del país, apelando a la responsabilidad social y educativa de estos ${ }^{10}$.

El presente desarrollo aportó algunos datos y descripciones sobre el inmenso trabajo de capacitación y generación de experiencias en comunicación y educación de la Defensoría del Público. Desde el 2012, estos proyectos y talleres se multiplicaron exponencialmente en varias provincias de la Argentina, promoviendo los derechos de las audiencias y el derecho a la comunicación desde un accionar ejemplar en el mundo. Sin embargo, como consecuencia de las nuevas políticas comunicacionales adoptadas en el país (Mastrini G. y Becerra, M., 2017) ${ }^{11}$, desde diciembre del 2016 hasta septiembre del 2018 el organismo estuvo acéfalo y actualmente se encuentra intervenido, por lo que conserva un equipo de trabajo mínimo, un presupuesto acotado y no ha podido abrir nuevas áreas de trabajo.

\section{HORA LIBRE, UNA APUESTA A LA ARTICULACIÓN Y TRANSFORMACIÓN SOCIAL}

El programa televisivo Hora libre surgió en el 2017 a partir del trabajo que venían realizando el Área de Capacitación y Acompañamiento de la Defensoría del Público y el programa REC (Red-Escuela-Comunicación) ${ }^{12}$. Estas dos organizaciones empezaron a

10 Desde hace más de seis años, la Defensoría del Público presenta los resultados de los monitoreos de noticias a organismos públicos, educativos, organizaciones sociales y referentes de empresas privadas de medios de comunicación nacionales. Con esto se busca debatir sobre la construcción de las noticias y los estereotipos discriminatorios en ellas, y así promover capacitaciones que deriven en la modificación de estos sentidos sociales circulantes, principalmente en los medios hegemónicos. Para mayor información sobre los monitoreos se puede acceder al sitio web de la Defensoría del Público de Servicios de Comunicación Audiovisual.

11 En diciembre del 2015, el Poder Ejecutivo Nacional, presidente Mauricio Macri, emitió una serie de decretos de necesidad y urgencia (267/15 y 1340/16, entre los más relevantes), que atentaron contra el espíritu democrático de la Ley SCA. Se abrió así una nueva etapa en Argentina que dio paso a políticas por las que el Estado habilitó el crecimiento de la concentración infocomunicacional y congeló el acceso y financiamiento a medios comunitarios y espacios para la pluralidad de voces (Mastrini y Becerra, 2017).

12 REC (Red-Escuela-Comunicación) es un programa educativo perteneciente a la Gerencia Operativa de Inclusión Educativa, de la Dirección General de Estrategias para la Educabilidad, Subsecretaría 
pensar un espacio en televisión donde niños, niñas y jóvenes de escuelas primarias y secundarias de la Ciudad Autónoma de Buenos Aires puedan participar de la producción audiovisual ${ }^{13}$. La televisora con la que se propuso realizar los primeros programas fue Barricada $\mathrm{TV}^{14}$, a la que la defensoría había brindado previamente talleres sobre derechos comunicacionales para niños, niñas y jóvenes.

Entre estos tres actores acordaron que trabajarían un formato televisivo que logre incluir géneros de entretenimiento, educación y cultura, a fin de habilitar un espacio donde los jóvenes puedan sentirse libres de expresarse y estén acompañados por sus escuelas. Así nació el concepto de Hora libre.

Cada programa estuvo realizado por una escuela diferente, duró aproximadamente una hora y se organizó en tres bloques, cada uno de los cuales desarrolló una o dos secciones preestablecidas por la Defensoría del Público. Algunas de las secciones trabajadas fueron: "¿Qué hay de ESI en eso?" —vinculada a las temáticas de educación sexual integral-, "Un lugar en mi escuela", “Nuestro barrio", "Momento Youtuber", “Momento artístico" y "Booktubers", basada en lecturas escolares y extraescolares de libros en papel o digitales.

Asimismo, se pensaron algunos ejes referidos a la comunicación y la educación: el uso de las tecnologías en la escuela y la producción autónoma de los estudiantes, el rol de los docentes, las actividades escolares vinculadas a la comunicación y los medios, en especial los medios comunitarios, alternativos y populares (Vinelli y Rodríguez Esperón, 2004).

Durante el último trimestre de 2017, se realizaron seis programas de televisión en Barricada TV junto con escuelas públicas de gestión estatal. Estas no necesariamente eran cercanas territorialmente al canal ${ }^{15}$, sino que la selección se relacionó con la predis-

de Equidad Educativa del Ministerio de Educación del Gobierno de la Ciudad de Buenos Aires. Surge en el 2006 con el objetivo de coordinar el trabajo de las escuelas con orientación en comunicación y con la difusión del trabajo radial que se realiza en las escuelas de la ciudad. Desde entonces, su intervención se ha extendido a escuelas primarias y secundarias de todo el distrito

13 En Argentina existen grupos audiovisuales de cine que trabajan en la producción audiovisual comunitaria con niños, niñas y jóvenes del país, principalmente en la generación de microrrelatos. Algunos de estos grupos son Laboratorio Audiovisual Comunitario (LAC) y Cine en Movimiento, cuyas producciones pueden verse en sus respectivos canales de YouTube.

14 Barricada TV es un canal de televisión alternativo y popular, que transmite por el canal 32.1 de la Televisión Digital Abierta de la ciudad de Buenos Aires. Funciona desde el 2009 en la fábrica IMPA, Almagro, una empresa recuperada por sus trabajadores.

15 Desde las escuelas y la defensoría se solicitó al gobierno de la ciudad que cubriera los gastos de traslados, para que chicas y chicos sin recursos económicos pudieran asistir al canal a grabar el programa. 
posición de directivos y docentes a llevar adelante el proyecto ${ }^{16}$. De estas experiencias, Cecilia Uriarte, trabajadora del organismo, recuerda:

Se debatió mucho cuál iba a ser el rol de las y los adultos en este proceso, con la idea de que la mediación adulta tenga la menor presencia posible, cómo iba a ser la conducción, si la iban a hacer los chicos y/o los adultos. Hasta que finalmente acordamos que tenía que haber un conductor/a pero su rol iba a ser de andamiaje, acompañamiento, facilitador de la palabra. Es un desafío no poner la mirada adulta y que los chicos y las chicas puedan tener un espacio de expresión. (Comunicación personal, 27 de octubre del 2018)

El relato de la trabajadora permite no solo pensar en los niños, niñas y jóvenes como sujetos de derecho a la comunicación sino, además, como "involucrados y decisores" -en términos de Roger Hoggart-, de los contenidos a trabajar en el programa de TV, aunque no así gestores ni organizadores del mismo (Edupas, 2006).

En muchos casos fueron necesarias capacitaciones a docentes de diversas áreas, y acercar a las escuelas materiales didácticos y herramientas comunicacionales que les permitiesen abrir espacios comunicacionales nuevos y diferentes en sus clases. Esto le valió al proyecto un doble esfuerzo, puesto que se invitó a los docentes a repensar su rol, su formación docente, y proponerse un trabajo dialógico y que excediese al currículum escolar. Todo un desafío que, como describen Paulo Freire e Ira Shor (2014), implica superar diversas variables y no temer a la osadía de la tarea docente:

Hay mucha presión para que se enseñe de esta forma tradicional. En primer lugar porque es familiar y ya está funcionando, aunque no dé resultados en clase. En segundo lugar, porque, al apartarte del programa oficial, puedes ser considerado un rebelde o un disconforme, y estar sujeto a algo que puede ir desde pequeños inconvenientes hasta el despido. (Freire y Shor, 2014)

Esta primera experiencia audiovisual recuperó lo central de aquella radial y propuso romper con lo que se espera del conocimiento escolar, abriendo espacios de educomunicación dialógica (Barbas Coslado, 2012) para que niños, niñas y jóvenes puedan ejercer su derecho educativo y comunicacional.

16 Muchas de estas escuelas habían trabajado previamente con la experiencia radial de REC y/o conocían los requisitos para trabajar con medios en las escuelas, entiéndase autorizaciones para registro y difusión de imagen de los chicos, salidas escolares, trabajo con herramientas audiovisuales y nuevas tecnologías de la información y de la comunicación. Las escuelas que participaron en el primer ciclo fueron: las primarias $n .^{\circ} 18$ provincia de La Rioja y n. ${ }^{\circ} 19$ República Italiana del distrito escolar 4; la escuela media $n .^{\circ} 2$ del distrito escolar 19, Arturo Jauretche; la escuela media . $^{\circ} 1$ del distrito escolar 7; la escuela técnica Raggio y la escuela media $n .^{\circ} 2$ del distrito escolar 17 , Rumania. 


\section{REFLEXIONES EN TORNO AL PROGRAMA HORA LIBRE REALIZADO EN MENDOZA}

En el 2018, desde la Defensoría del Público, se buscó reproducir la experiencia de Hora libre en la provincia de Mendoza, a 1080 km de la Ciudad Autónoma de Buenos Aires, y en coordinación con Giramundo Televisión Comunitaria. Si bien el modelo de programa propuesto fue el mismo, la experiencia adoptó características territoriales, en función del trabajo previo de la televisora comunitaria ${ }^{17}$, la formación docente y los contenidos trabajados en las escuelas convocadas para el proyecto, que se describirán brevemente a continuación.

El territorio donde esta experiencia tuvo lugar fue el del canal comunitario Giramundo TV, ubicado en la localidad de San José, departamento de Guaymallén, uno de los más densamente poblados de la provincia de Mendoza y cuya característica socioeconómica es la creciente desigualdad social ${ }^{18}$. Las escuelas zonales son muy desiguales y acceden a ellas niños, niñas y jóvenes de sectores vulnerables y, en muchos casos, poblaciones migrantes.

Desde el 2009, la televisora transmite las 24 horas del día por canal 34 de aire (gratuito), uno de los siete canales locales que actualmente operan en el Gran Mendoza. Los canales de televisión a los que la población puede acceder de manera gratuita son público-estatales o privados nacionales o internacionales y sus contenidos son mayoritariamente producciones generadas en la Ciudad Autónoma de Buenos Aires. Los

17 Algunas experiencias previas fueron: Cano TV (2012), un informativo de media hora desde el cual chicos y chicas de séptimo grado (de 11 a 12 años aproximadamente) de la Escuela Cano pudieron dar a conocer lo trabajado en el taller de salud organizado por la Consultoría de Salud Popular de Guaymallén en la escuela primaria; talleres de producción audiovisual con técnica stop-motion para jóvenes de la escuela secundaria Abelardo Vázquez (2014), centrados en la prevención de violencia de género y violencia escolar, ofrecidos de manera gratuita y realizados en coordinación con las docentes de la materia Comunicación Social de la escuela; y un taller de producción audiovisual en la escuela técnica San José (2015), dentro del programa Centro de Actividades Juveniles (CAJ), en el que participaron cerca de 12 estudiantes de diferentes cursos. Asimismo, el canal trabajó con los centros educativos Jorge A. Paschuan 3-415, del departamento de Las Heras (2013), y Belgrano Educador 3-408, de la ciudad de Mendoza (2014), ambos de secundaria para jóvenes y adultos. El espacio de la televisora se abrió desde el 2009 para que docentes de todos los niveles realicen visitas guiadas, entrevistas y presentaciones artísticas en vivo con sus estudiantes, centradas en el derecho a la comunicación y la autogestión del espacio. Otras experiencias son los proyectos de Extensión Universitaria Mauricio López, de la Universidad Nacional de Cuyo, y la apertura del canal como centro de prácticas profesionalizantes a la Tecnicatura en Producción Audiovisual de la Universidad Nacional de Cuyo.

18 Se trabaja en los distritos de San José Belgrano, Bermejo y Pedro Molina, con un total de 74194 habitantes, según el Censo del 2010. Es una zona urbana de alta densidad poblacional, que tiene una fuerte desigualdad en cuanto a las condiciones habitacionales, acceso a servicios y consumo de bienes culturales y sociales. Además, evidencia un sector de la población que, si bien vive en zona urbana, desarrolla sus actividades laborales en la zona este, donde se encuentra el área productiva del departamento. En cuanto al acceso al sistema educativo y medios de comunicación, esta zona de Guaymallén cuenta con una amplia oferta educativa, con una radio comunitaria, La Mosquitera, y dos espacios de producción audiovisual comunitaria: GiraMundo TV y La Mosquitera Audiovisual. 
ciudadanos que desean acceder a mayores canales y contenidos deben abonar un servicio de cable, servicios de internet o plataformas online prepagas.

En lo que respecta a la producción de contenidos destinados a niños, niñas y jóvenes en la provincia de Mendoza, las televisoras de acceso gratuito (canales de aire) transmiten programas principalmente enlatados (preproducidos, no en vivo) y la participación de niños y jóvenes es mínima o nula. Únicamente en el canal estatal provincial (Acequia TV) tuvo lugar un programa infantil en el que niños y niñas de la provincia fueron protagonistas como narradores de historias producidas por ellos mismos.

El diseño curricular de la provincia de Mendoza cuenta con clases de comunicación social obligatorias en el segundo año de secundaria, a cargo de licenciados, técnicos y profesores en comunicación. En la asignatura se acercan contenidos como sistemas de comunicación, medios de comunicación clásicos y digitales, la imagen como concepto, publicidad, propaganda, entre otros. El programa está diagramado para trabajar principalmente desde una perspectiva crítica de los medios (Orozco Gómez, 1986), pero en ninguna instancia se aborda la comunicación como un derecho. En este sentido, se adeuda una revisión crítica sobre el currículum escolar en relación con el cambio de paradigma comunicacional ${ }^{19}$.

\section{Surgimiento y organización del proyecto}

Durante el primer trimestre de 2018 se realizó una reunión entre Giramundo TV Comunitaria y la Defensoría del Público en la que se establecieron las líneas de trabajo y se acordó el carácter local que tomaría el proyecto. Se propició la recuperación de las experiencias previas del canal en lo que respecta a la articulación con escuelas, pero se duplicó la apuesta. El magacín Hora libre se centraría en las producciones de niños, niñas y jóvenes y el canal realizaría las tareas vinculadas al soporte técnico y la coordinación con las instituciones. Así, la defensoría preparó un proyecto escrito y el canal inició un trabajo de articulación con las escuelas de la zona ${ }^{20}$.

En un primer acercamiento, la coordinación con las escuelas pareció sencilla. Sin embargo, frente a los requisitos escolares de trabajo curricular, permisos de difusión de

19 El análisis del currículo de estas materias es desarrollado en el proyecto de investigación en comunicación y educación de la Universidad Nacional de Cuyo, "La enseñanza de la comunicación en la escuela secundaria. Resignificación del currículum desde la educación mediática crítica (20162017)". Los resultados aún están en proceso de publicación.

20 Se trabajó con el quinto año de la Escuela del Magisterio de la Universidad Nacional de Cuyo, orientación en comunicación; dos escuelas secundarias públicas de gestión estatal, Cacique Guaymallén y Técnica San José, las cuales tienen la materia de comunicación en segundo y cuarto año respectivamente; una escuela primaria pública de gestión estatal, Escuela Vuelta de Obligado, en la que se desarrolla un taller de radio; y el colegio Alberto Schweitzer de la Iglesia Metodista de Mendoza, de nivel secundario que incluye comunicación en su currículo. 
imagen y salidas escolares, sembraba muchas dudas entre los docentes que no sabían cómo entrelazar la cultura escolar con la mediática poniendo el acento en los intereses de los educandos (Huergo, 1997).

Es por ello que, desde el canal, se tomaron dos estrategias. Por un lado, solicitar a la Defensoría del Público su vínculo directo con las escuelas para la presentación del proyecto. Por el otro, se solicitó la realización de un taller de capacitación sobre derechos comunicacionales de niños, niñas y jóvenes, en el que se trabajaría este eje vinculado a la propuesta de Hora libre, y en el que participarían tanto docentes como estudiantes (ver figura 1), a fin de propiciar el intercambio y reforzar la idea del protagonismo de niños, niñas y jóvenes en esta experiencia.

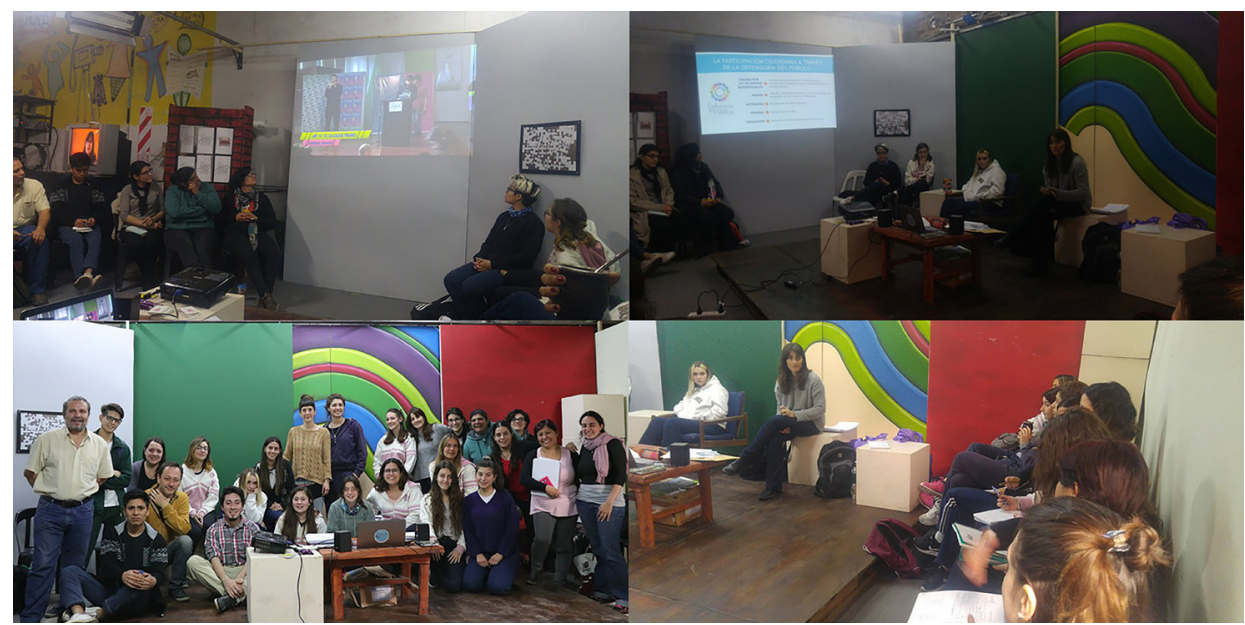

Figura 1. Taller de derechos comunicacionales de niños, niñas y jóvenes

Fuente: Archivo fotográfico de Giramundo Televisión Comunitaria, Mendoza, Argentina

¿De qué modo la escuela puede propiciar espacios para el ejercicio del derecho a la comunicación? ¿Desde qué abordaje se trabajaría la comunicación en el aula? Hora libre se acercó, así como una apuesta, a crear instancias de educomunicación en las escuelas. Jorge Huergo (en Arena Fernández, 2012, p.13) explica que el desafío de este campo consiste no solo en utilizar los medios como herramientas para el proceso educativo, sino, además, abrir espacios de producción que desencadenen "procesos de formación subjetiva", lo que implica ejercer "otro tipo de lectura sobre la escuela, sobre la institución escolar y sobre el trabajo de los educadores".

Durante la capacitación, se invitó especialmente a los docentes a proponer actividades en el aula que excedan el diseño curricular y la reflexión crítica sobre los medios masivos, generando actividades que vehiculicen las producciones para el programa 
pero reflexionando sobre lo que los estudiantes querían contar y cómo. Para ello, se brindó asistencia desde la Defensoría del Público distribuyendo materiales didácticos diseñados por el organismo ${ }^{21}$. Muchas de las actividades propuestas en estos manuales están pensadas para trabajar desde diversas asignaturas sobre estereotipos (en los medios y socialmente cristalizados). El material plantea no solo el análisis crítico de discursos mediáticos para identificar las representaciones sociales discriminatorias, sino que desarrolla ejercicios que habiliten la reconstrucción de estas representaciones desde las realidades sociales de cada aula. Al respecto, Cora Gamarnik (2009) afirma:

La intervención educativa tanto en el ámbito formal como no formal puede lograr cambios a gran escala. Conocer y analizar en las escuelas el funcionamiento de los estereotipos ayudaría a formar ciudadanos más alertas. Hay varias preguntas y estrategias didácticas que pueden utilizarse para esto: ¿Las imágenes que se presentan como representativas de ciertos grupos son las que ese grupo hubiese elegido para representarse a sí mismo? ¿Quién decide quién habla por quién? ¿Quién se beneficia con el uso de estereotipos negativos de determinados grupos sociales? Numerosas actividades en este sentido pueden ayudar a la reflexión, comprensión e inicio de un cambio de actitudes. (Gamarnik, 2009, p. 9)

Durante el taller, se invitó tanto a adultos como jóvenes a analizar los resultados del último informe de los monitoreos de medios y se debatió acerca del lugar que niños, niñas y jóvenes tienen (o no) en los programas televisivos. Asimismo, se analizaron series de ficción y se visibilizaron los estereotipos de niños y jóvenes puestos en circulación en ellas. Es interesante destacar la capacidad crítica con la que los estudiantes asistentes al taller pudieron realizar estos ejercicios y su llamado de atención sobre cómo las ficciones (principalmente telenovelas) toman a un sector de jóvenes de la Ciudad Autónoma de Buenos Aires como el modelo homogeneizador de las chicas y los chicos de toda la Argentina, no respetando la diversidad sociocultural del país.

Estos disparadores invitaron a los estudiantes a pensar cómo presentarse y qué contar desde su propia perspectiva y realidad. A continuación, relataremos brevemente cómo fue este proceso y qué reflexiones se dieron al respecto.

\section{Preproducción y realización de los programas}

Durante el mes de noviembre del 2018, se grabaron cuatro programas en las instalaciones de Giramundo TV Comunitaria con estudiantes de cinco escuelas diferentes. Para asistir a estas instancias, cada grupo debía previamente preproducir cápsulas para las secciones acordadas, es decir, microrrelatos audiovisuales que visibilicen el trabajo sobre las temáticas. Asimismo, se solicitó a las escuelas que cumplan con las

21 Materiales disponibles en el sitio web de la Defensoría del Público de Servicios de Comunicación Audiovisual. 
autorizaciones de difusión de imagen correspondientes a cada niño, niña o joven que había sido o sería filmado. Tanto las familias como los niños y jóvenes registraban su conformidad para salir en la TV.

Así, durante tres meses se realizaron los rodajes de las cápsulas en cada escuela, con trabajo de estudiantes y docentes tanto en horario escolar como extraescolar. Algunas secundarias fueron más autónomas en sus trabajos puesto que los jóvenes tenían manejo de herramientas audiovisuales y los docentes acompañaron en la construcción de los relatos para las secciones. En cambio, en las otras escuelas no pudieron organizar actividades para la producción audiovisual, por lo que, a un mes de realizar los programas, el canal de televisión intervino acompañando el trabajo de producción y colaborando en la organización del relato y manejo de cámaras y teléfonos celulares.

Esta demora o desorganización en el trabajo se puede evaluar desde dos aspectos: la falta de manejo de herramientas audiovisuales o la imposibilidad de incluir una propuesta no escolar en los tiempos y dinámicas escolares. Con respecto al primer punto, podemos pensar que, si las nuevas generaciones son efectivamente nativos digitales, las producciones podrían haberse realizado con mayor facilidad. Sin embargo, algunos estudiantes de la escuela secundaria manifestaron no poder hacer el trabajo porque sus celulares o netbooks (adquiridas a través del programa Conectar Igualdad ${ }^{22}$ ) no soportaban la edición de video o porque no podían utilizar las computadoras portátiles que les facilitaba la escuela fuera del horario institucional. Otros contaban con áreas de tecnología en la escuela y con equipamiento para registrar videos (netbooks más nuevas, cámaras de fotos, tablets) sin embargo, no se utilizaron para la producción en el horario escolar.

Las diversas situaciones que se dieron en relación con el uso de las NTIC nos permiten preguntarnos qué rol cumplen estas en la escuela y qué concepción de la comunicación prevalece en algunas instituciones. Sobre este punto, el profesor e investigador Ángel Barbas Coslado (2012, p. 163) afirma que es habitual encontrar "algunos planes escolares que incorporan los recursos digitales a los procesos educativos, sin considerar los planteamientos emancipadores, críticos o dialógicos en el uso de los medios".

22 Conectar Igualdad es un programa enmarcado en la Ley de Educación Nacional (Ley 26206, 2006), iniciado en el 2010, que consiste en la entrega de una computadora tipo classmate por estudiante y a sus docentes en las escuelas públicas secundarias de gestión estatal del país. En sus inicios, el programa abrió la posibilidad de trabajar contenidos digitales e introducir nuevas formas de enseñar y aprender con las nuevas tecnologías de la información y de la comunicación (Lago Martínez, Marotias y Amado, 2012). Actualmente el programa se encuentra vigente pero desfinanciado, por lo que desde el 2017 no se han entregado más computadoras a los jóvenes. Se ha propiciado, desde el plan Aprender Conectados, dentro de Conectar Igualdad, el equipamiento escolar y la conectividad a internet a través de fibra óptica. 
Podemos citar otra situación que se visibilizó en Hora libre en relación con lo recientemente explicado. En la escuela primaria, las maestras de lengua y ciencias sociales tomaron las secciones para trabajar contenidos escolarizados, lo que derivó en el registro audiovisual de frases memorizadas de libros leídos en clase. Asimismo, se presentó y charló sobre un video producido por la profesora de teatro en el que se mostraron actividades de expresión inspiradas en la lectura del libro. Estas acciones dejaron fuera del proyecto la posibilidad de que los estudiantes puedan opinar sobre el material trabajado o comentar libros, revistas, blogs que les interesen y recomendarlos.

Para recuperar la perspectiva de los alumnos, durante la grabación del programa desde el canal y la defensoría se los invitó a dar su opinión sobre el libro, sobre la tarea de enseñar a leer (temática que trataba el libro escolar) y su relación con sus vivencias como estudiantes.

Algo similar sucedió con un grupo de una escuela secundaria. Para la presentación y análisis de un video en la sección de educación sexual integral (ESI), el profesor les había recomendado a los estudiantes qué decir respecto a la temática del video (violencia de género). Así, en el debate todos estuvieron de acuerdo en que ejercer violencia hacia las mujeres "está mal", pero cuando la conductora preguntó por qué pensaban eso, fue difícil de explicar (revisar video). Frente a la insistencia de la conductora, un estudiante pudo manifestar su desacuerdo y contar lo que pensaba su familia respecto de la ESI. La expresión del joven generó un debate muy enriquecedor que permitió revalorizar el espacio de la ESI en las escuelas y los chicos pudieron expresar sus posiciones diversas.

Al respecto, Cecilia Uriarte, referente de capacitación de la Defensoría del Público, comentó que se vivieron situaciones similares en la experiencia de Hora libre de Barricada TV y reflexionó: "A veces lo que se espera de la escuela genera mediaciones que terminan produciendo cosas excesivamente escolarizadas. ¿De qué manera es posible trabajar con los docentes para que no se sientan expuestos y superen esto?" (comunicación personal, 27 de octubre del 2018).

Las experiencias fundamentadas en concepciones instrumentalistas de la comunicación y las tecnologías no garantizan el ejercicio de derechos. Para hacerlo, es necesario un cambio de perspectiva pedagógica y la implementación de políticas públicas de distribución de tecnologías, acompañadas por capacitaciones, talleres, encuentros interinstitucionales y otras estrategias que desde los gobiernos escolares se puedan generar (Huergo, 1997).

Finalmente, recuperaremos un detalle no menor en la realización del programa: ¿Quién tiene la palabra? ¿Quién hace uso del micrófono? Tanto en la experiencia de Hora libre de Barricada TV, como en la de Giramundo TV estuvo presente este debate. En ambos casos, las organizaciones participantes decidieron que la conducción general sea realizada por un/a referente adulto/a vinculado al proyecto, pero con la consigna de abrir 
preguntas, vehiculizar la palabra y no interferir en las opiniones de los jóvenes. Para ello, se tuvo en cuenta el uso de un micrófono de mano solo para los estudiantes y un corbatero para el/la conductor/a. De este modo, se habilitaría la circulación de la palabra y se evitaría una perspectiva adultocéntrica, generalmente visible en los medios de comunicación hegemónicos, en los que el adulto tiene el micrófono y se lo acerca al niño o niña solo para que responda (ver figura 2).

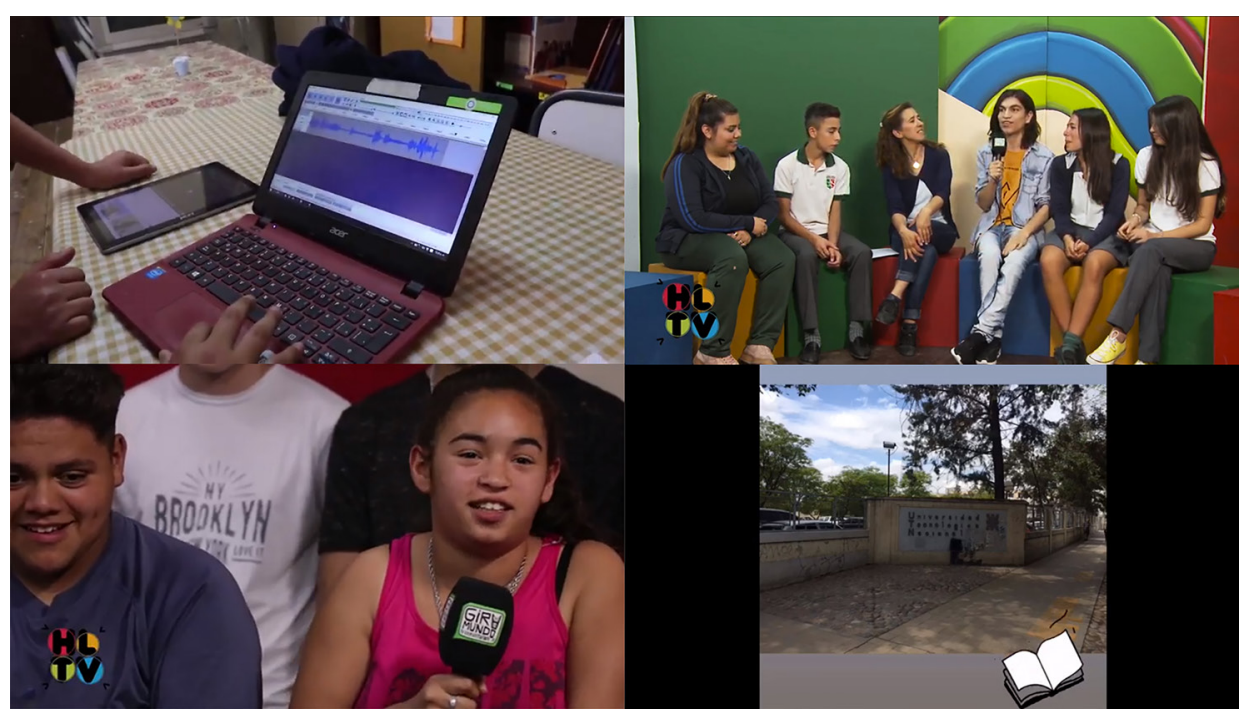

Figura 2. Programa en el piso y preproducciones de los jóvenes

Fuente: Archivo fotográfico de Giramundo Televisión Comunitaria, Mendoza, Argentina.

El relato del proceso de producción y realización de Hora libre en Mendoza nos invita a la reflexión sobre los espacios de expresión para que los chicos puedan ejercer su derecho a la comunicación. Pero, además, nos propone el desafío de pensar en clave de la educomunicación y preguntarnos "cómo estamos formando la subjetividad con los medios masivos, los medios comunitarios y cómo nos posicionamos como profesionales frente a eso" (Huergo como se citó en Arena Fernández, 2012, p. 13). 


\section{REFLEXIONES Y APORTES DESDE LA EXPERIENCIA}

Es porque conocemos las leyes de la reproducción por lo que tenemos alguna oportunidad de minimizar la acción reproductora de la institución escolar.

Bourdieu, 2008, p. 139

Desde la sanción de la Ley SCA (Ley 26522, 2009) en Argentina, diversas estrategias se pusieron en marcha para el cumplimiento de políticas destinadas al ejercicio del derecho a la comunicación y, desde este, al respeto hacia los derechos humanos en general. En el caso de los derechos de niños, niñas y jóvenes, ponemos en valor el rol de la Defensoría del Público como organismo que resguarda derechos por el estudio y monitoreo de medios, pero que se propone como articulador de actores e instituciones, y que apuesta a la capacitación como factor de transformación social.

La realización del programa televisivo Hora libre en la Ciudad Autónoma de Buenos Aires y en la provincia de Mendoza constituyen antecedentes únicos en la Argentina, donde medios de comunicación y escuelas cooperan en un trabajo colectivo para que chicos y chicas sean protagonistas en ello. Asimismo, estas experiencias se constituyen como una oportunidad para reflexionar sobre la relación entre comunicación y educación mediática en nuestro país, y como campo disciplinar que permite el análisis de dispositivos, situaciones, proyectos y experiencias donde la educación y la comunicación se relacionen desde una perspectiva superadora y transformadora de ambos campos (Arena Fernández, 2012).

Es por ello que, en la experiencia trabajada, entendemos que la articulación desde la defensoría con medios de comunicación comunitarios no es casual y no solo se vincula con el contexto político-comunicacional. La lógica de trabajo que estos medios llevan adelante abre la posibilidad de repensar procesos educativos desde la autogestión, cooperativos, solidarios, sin fines de lucro y con fines políticos (Vinelli y Rodríguez Esperón, 2004). En estos medios el minuto de aire no tiene precio y el tiempo invertido en capacitación, debate, intercambio, reflexión y educación es el motor para la acción (Pulleiro, 2012). Pero, además, en estos proyectos la tecnología es una herramienta de trabajo al servicio de la comunidad por lo que diversos usos sociales son posibles de abordar.

Cuando a lo largo del presente trabajo nos preguntamos por el rol de las nuevas tecnologías de la información y de la comunicación (NTIC) en la escuela, la experiencia de los medios comunitarios aportó una posible respuesta: la tecnología como condición de posibilidad, no como una herramienta comunicacional. Al decir de Fernando Reyes Matta 
(1989, p. 372), "Es la conciencia de lo que se quiere decir la que hace de esas tecnologías una posibilidad, una recuperación y un campo de acción en la perspectiva de la liberación del individuo y de la sociedad".

En la experiencia de Hora libre el rol de la escuela en relación con los medios de comunicación fue puesto en jaque. Se le propuso al sistema educativo dejar de ser el centro informativo y formativo en el uso de las nuevas tecnologías y abrirse a las formas de apropiarse de estas de los estudiantes. También se le propuso a la escuela aceptar los discursos y los consumos culturales de niños, niñas y jóvenes, y repensar el lugar de educadores y educandos dentro y fuera del aula (Freire, 2009).

El proceso de planificación, trabajo y realización del programa televisivo invitó a estudiantes, docentes, comunicadores, técnicos en audiovisual y especialistas a cuestionarse sus propios roles y lugares, a pensar productos nuevos que garanticen derechos que no consumen en cotidiano y a evaluar(se) como sujetos de derechos y productores antes que como espectadores y depositarios. Retomamos entonces la definición propuesta por Barbas Coslado (2012), quien entiende que la Educomunicación es:

proceso, movimiento, flujo de significados, acción creativa y re-creativa, construcción-deconstrucción-reconstrucción permanente de la realidad. Es, en suma, una forma de pedagogía crítica que concibe los procesos educativos, la comunicación, los medios y las tecnologías como herramientas de análisis y de acción para la comprensión y la transformación del mundo. (Barbas Coslado, 2012, p. 167)

La vivencia y sistematización de Hora libre fue analizada como una oportunidad para visibilizar lo que los medios hacen con las representaciones de niños, niñas y jóvenes. Pero fundamentalmente, para "asumir las complejas transformaciones que atraviesan nuestras sociedades. Ya que los verdaderos cambios hoy no son en la tecnología sino en la sociedad, o mejor en la sensibilidad (el sentir implica tanto los sentidos como los sentimientos) de la gente de común" (Martín Barbero como se citó en Massoni, 2016, p. 11).

La puesta en situación de estos actores para generar un producto nuevo y diferente (no solo en términos del programa televisivo) constituye un ejercicio de educomunicación dialógica, donde el diálogo se torna inseparable de la práctica educativa y de los procesos de aprendizaje interdisciplinarios y colectivos (Barbas Coslado, 2012). Un ejemplo de esto es la visibilización de reclamos e intereses de los jóvenes en sus familias e instituciones educativas a partir del programa, y la incorporación del derecho a la comunicación en los currículos de las materias de comunicación que articularon con los proyectos, lo que implica no solo un agregado sino un cambio de posición político-pedagógica con los estudiantes.

De este modo, consideramos que la generación de contextos que propician el debate público por la democratización de las comunicaciones y las acciones ciudadanas respaldadas por políticas de Estado colaboran con la transformación de las prácticas y 
perspectivas de trabajo solo si ellas son puestas en diálogo y acción. Al respecto, Jesús Martín Barbero en La educación desde la comunicación, recuerda: "Dialogar es descubrir en la trama de nuestro propio ser la presencia de los lazos sociales que nos sostienen. Es echar los conocimientos a una posesión colectiva, comunitaria, del mundo" (Martín Barbero, 2002, p. 35).

\section{REFERENCIAS}

Arena Fernández, A. (2012). Educomunicación, ciudadanía y medios escolares: entrevista con cinco investigadores latinoamericanos. Contextos, (1), 11 - 18. Recuperado de https://www.researchgate.net/publication/322480675_Educomunicacion_ ciudadania_y_medios_escolares_entrevista_con_cinco_investigadores_ latinoamericanos

Barbas Coslado, A. (2012). Educomunicación: desarrollo, enfoques y desafíos en un mundo interconectado. Foro de Educación, (14), 157-175. Recuperado de http:// www.redalyc.org/pdf/4475/447544618012.pdf

Bourdieu, P. (2008). Capital cultural, escuela y espacio social. Buenos Aires: Siglo XXI Editores.

Defensoría del Público de Servicios de Comunicación Audiovisual. (2018). Informe: 5 años de monitoreo de noticias (2013-2017). Ciudad Autónoma de Buenos Aires. Recuperado de https://defensadelpublico.gob.ar/resultado-delanalisis-de-cinco-anos-de-monitoreos-de-programas-noticiosos

Edupas. (2006). Cuadernillo 3: Participación de niñas, niños y adolescentes. Argentina: Unicef.

Espada, A. (2017). Una oportunidad perdida: la ley y los medios sin fines de lucro. En M. Becerra, Medios en Guerra. Balance, crítica y desguace de las políticas de comunicación 2003-2016, (pp. 79-114). Buenos Aires: Editorial Biblios.

Freire, P. (2002). Pedagogía del oprimido. Buenos Aires: Siglo XXI Editores.

Freire, P. (2009). Pedagogía de la autonomía. Saberes necesarios para la práctica educativa. Buenos Aires: Siglo XXI Editores.

Freire, P. y Shor, I. (2014). Miedo y osadía. La cotidianidad del docente que se arriesga a practicar una pedagogía transformadora. Buenos Aires: Ed. Siglo XXI.

Gamarnik, C. (2009). Estereotipos sociales y medios de comunicación: un círculo vicioso. Ciencias de la Comunicación. Buenos Aires: Universidad de Buenos Aires. Recuperado de http://sedici.unlp.edu.ar/bitstream/handle/10915/33079/Docu mento_completo.pdf?sequence $=1$ 
Graziano, M. (1980). Para una definición alternativa de la comunicación. Revista Ininco, 1(1), 71.

Hall, S. (2003). Introducción: ¿Quién necesita 'identidad'? En Cuestiones de Identidad Cultural (pp. 13-39). Buenos Aires: Amorrortu.

Huergo, J. (2015). La educación y la vida. Un libro para maestros de escuela y educadores populares. La Plata: Ediciones de Periodismo y Comunicación, Universidad Nacional de La Plata. Facultad de Periodismo y Comunicación Social.

Huergo, J. (. (1997). Comunicación/Educación. Ámbitos, prácticas y perspectivas. La Plata: Ediciones de Periodismo y Comunicación, Universidad Nacional de La Plata.

Lago Martínez, S., Marotias, A. y Amado, S. (2012). Inclusión digital en la educación pública argentina. El Programa Conectar Igualdad. Revista Educación y Pedagogía, 24(62), 205-218. Recuperado de http://aprendeenlinea.udea.edu.co/revistas/index.php/ revistaeyp/article/view/14204/12547

Lamas, E. y Lewin, H. (1995). Aproximación a las radios de nuevo tipo: tradición y escenarios actuales. Causas y azares, (2).

Ley 26.522 de Servicios de Comunicación Audiovisual. (10 de octubre del 2009) Honorable Congreso de la Nación Argentina, Ciudad Autónoma de Buenos Aires, Argentina.

Ley 26061 de Protección Integral de los Derechos de Niños, Niñas y Adolescentes. (28 de septiembre del 2005). Infoleg (Información Legislativa), Ministerio de Justicia y Derechos Humanos, Buenos Aires, Argentina.

Ley 26.206 de Educación Nacional. (14 de diciembre del 2006). Infoleg (Información Legislativa), Ministerio de Justicia y Derechos Humanos, Presidencia de la Nación, Buenos Aires, Argentina.

Martín Barbero, J. (1996). Heredando el futuro. Pensar la educación desde la comunicación. Nómadas, (5). Recuperado de http://www.redalyc.org/pdf/1051/105118998 002.pdf

Martín Barbero, J. (2002). La educación desde la comunicación. Buenos Aires: Editorial Norma.

Massoni, S. (2016). Avatares del comunicador complejo y fluido. Del perfil del comunicador social y otros devenires. Quito, Ecuador: Ediciones Ciespal.

Mastrini G. y Becerra, M. (2017). Medios en guerra. Balance, crítica y desguace de las políticas de comunicación 2003-2016. Buenos Aires: Editorial Biblios.

Mastrini, G. y De Charras, D. (2004). Veinte años no es nada: del NOMIC a la CMSI. Universidad Nacional de Buenos Aires, Cátedra Políticas y Planificación de la Comunicación, 
Congreso IAMCR 2004, Porto Alegre, Brasil. Recuperado de www.catedras.fsoc. uba.ar/mastrini/investigaciones/mastrini_decharras.doc

Mata, M. (2006). Comunicación y ciudadanía. Problemas teórico-políticos de su articulación. Fronteiras - estudos midiáticos, 8(1), 5 - 15. Recuperado de http:// revistas.unisinos.br/index.php/fronteiras/article/view/6113/3289

Mata, M. C. (1988). Comunicación popular: de la exclusión a la presencia. Córdoba: Mimeo.

Morduchowicz, R. (1999). La educación para los medios es una educación para la democracia. Comunicar, (13), 117-122. Recuperado de https://dialnet.unirioja.es/ descarga/articulo/229978.pdf

Orozco Gómez, G. (1986). Computadoras y educación escolar: ¿una vinculación inevitable? (Inegi, Ed.) Comunidad informática, (25).

Pulleiro, A. (2012). La radio alternativa en América Latina. Experiencias y debates desde los orígenes hasta el siglo XXI. Buenos Aires: Cooperativa Gráfica El Río Suena.

Reguillo Cruz, R. (2000). Entre la insumisión y la obediencia. Biopolítica de los cuerpos jóvenes. En Emergencia de culturas juveniles. Estrategias del desencanto. (pp. 75-95). Buenos Aires: Norma.

Reyes Matta, F. (1989) La comunicación transnacional y la respuesta alternativa. En Simpson Grinberg, M. (comp.), Comunicación alternativa y cambio social. México, Premiá.

Rossi, D. (2016). Acceso y participación en el nuevo siglo. Limitaciones de la política, condicionamientos de los conglomerados. CECSO, UBA. Recuperado de http:// politicasyplanificacion.sociales.uba.ar/wp-content/uploads/sites/121/2014/07/ accesoyparticipacion2016.pdf

Sel, S. (2010). Actores sociales y espacio público. Disputas por la Ley de Servicios de Comunicación Audiovisual en Argentina. En S. Sel, Políticas de comunicación en el capitalismo contemporáneo. América Latina y sus encrucijadas (pp. 183 - 210). Buenos Aires: Clacso.

Vinelli, N. y Rodríguez Esperón, C. (2004). Contrainformación. Medios alternativos para la acción política. Buenos Aires: Ediciones Continente. 
